\title{
Video Article \\ A Novel High-resolution In vivo Imaging Technique to Study the Dynamic Response of Intracranial Structures to Tumor Growth and Therapeutics
}

\author{
Kelly Burrell ${ }^{1}$, Sameer Agnihotri ${ }^{1}$, Michael Leung ${ }^{2}$, Ralph DaCosta ${ }^{2}$, Richard Hill ${ }^{2}$, Gelareh Zadeh ${ }^{3}$ \\ ${ }^{1}$ Brain Tumor Research Centre, Hospital for Sick Children, Toronto Medical Discovery Tower \\ ${ }^{2}$ Ontario Cancer Institute, Princess Margaret Hospital \\ ${ }^{3}$ Neurosurgery, Toronto Western Hospital
}

Correspondence to: Kelly Burrell at Kelly.burrell@sickkids.ca

URL: https://www.jove.com/video/50363

DOI: doi: $10.3791 / 50363$

Keywords: Cancer Biology, Issue 76, Medicine, Biomedical Engineering, Cellular Biology, Molecular Biology, Genetics, Neuroscience, Neurobiology, Biophysics, Anatomy, Physiology, Surgery, Intracranial Window, In vivo imaging, Stereotactic radiation, Bone Marrow Derived Cells, confocal microscopy, two-photon microscopy, drug-cell interactions, drug kinetics, brain, imaging, tumors, animal model

Date Published: 6/16/2013

Citation: Burrell, K., Agnihotri, S., Leung, M., DaCosta, R., Hill, R., Zadeh, G. A Novel High-resolution In vivo Imaging Technique to Study the Dynamic Response of Intracranial Structures to Tumor Growth and Therapeutics. J. Vis. Exp. (76), e50363, doi:10.3791/50363 (2013).

\section{Abstract}

We have successfully integrated previously established Intracranial window (ICW) technology ${ }^{1-4}$ with intravital 2-photon confocal microscopy to develop a novel platform that allows for direct long-term visualization of tissue structure changes intracranially. Imaging at a single cell resolution in a real-time fashion provides supplementary dynamic information beyond that provided by standard end-point histological analysis, which looks solely at 'snap-shot' cross sections of tissue.

Establishing this intravital imaging technique in fluorescent chimeric mice, we are able to image four fluorescent channels simultaneously. By incorporating fluorescently labeled cells, such as GFP+ bone marrow, it is possible to track the fate of these cells studying their long-term migration, integration and differentiation within tissue. Further integration of a secondary reporter cell, such as an mCherry glioma tumor line, allows for characterization of cell:cell interactions. Structural changes in the tissue microenvironment can be highlighted through the addition of intra-vital dyes and antibodies, for example CD31 tagged antibodies and Dextran molecules.

Moreover, we describe the combination of our ICW imaging model with a small animal micro-irradiator that provides stereotactic irradiation, creating a platform through which the dynamic tissue changes that occur following the administration of ionizing irradiation can be assessed.

Current limitations of our model include penetrance of the microscope, which is limited to a depth of up to $900 \mu \mathrm{m}$ from the sub cortical surface, limiting imaging to the dorsal axis of the brain. The presence of the skull bone makes the ICW a more challenging technical procedure, compared to the more established and utilized chamber models currently used to study mammary tissue and fat pads ${ }^{5-7}$. In addition, the ICW provides many challenges when optimizing the imaging.

\section{Video Link}

The video component of this article can be found at https://www.jove.com/video/50363/

\section{Introduction}

A better understanding of the structural and biological changes that arise in the brain in response to various pathologies, and therapeutic interventions is critical for improving treatment strategies. However, one of the current challenges in studying these structural and biological changes, particularly regarding the intracranial pathologies, is the inaccessibility of the tissue and the inability to study the temporal evolution and dynamic progression of changes in an in vivo setting. The generation of the "window" technology has previously proved successful in examining soft tissue changes through tumor development ${ }^{5-7}$. Development of ICW models proves to be more technically challenging, due to the necessity to remove the skull bone without damaging on instigating infection in the underlying cerebral tissue. Previous papers have attempted to thin the skull to visualize the tissue ${ }^{8-10}$ however, to produce high resolution clear images full removal of the skull bone is required ${ }^{11}$. Repeat long-term imaging (30 days+) has only recently become a feasible option through imaging ${ }^{1,11}$, previously shorter time frames have been studied ${ }^{5}$.

Over the past decade a major goal has been to elucidate the origin of neo-vasculature following pathological stimuli, in particular in response to tumor formation and progression, to provide novel therapeutic targets for the treatment of tumors. Much controversy remains around the source of new vasculature in the brain during tumor development or following radiation. Traditionally vascularization has been considered to occur through angiogenesis, a process by which new vessels form from the sprouting of pre-existing vessels ${ }^{12}$. More recent studies however, suggest that the previously assumed embryonic process of vasculogenesis may be playing a more considerable role in the formation of pathological vasculature. Vasculogenesis involves the recruitment of the adult angioblast counterparts from the bone marrow which in turn are then directly involved in the formation of new vessel endothelium ${ }^{12-14}$. Accumulating evidence indicates that endothelial precursor cells are mobilized from the bone marrow to initiate de novo vessel formation in response to oncogenic mediators ${ }^{15-17}$. However, these studies provide contradictory 
evidence for the direct contribution of these bone marrow derived cells (BMDCs) to vessel endothelium with percentage contribution varying with the type of pathological stimulus and response to treatment ${ }^{18-21}$.

Therefore, establishing reproducible experimental methods that allow high-resolution intravital imaging for repeated long-term study of the process of BMDC integration into tumor vasculature and in response to therapy is invaluable. Standard histological techniques fail to provide the dynamic information required to determine the long-term survival, differentiation and integration of the cells that give rise to neovasculature and therefore cannot demonstrate definitively the mechanisms of cell interaction.

We have shown using our experimental approach that there is a varying degree of BMDC recruitment following both intracranial ionizing radiation and tumor growth, a recruitment that is however, pathology site specific and not an invasion of the entire intracranial tissue ${ }^{11,22}$. We have shown that the recruitment follows a time sensitive pattern demonstrated by the repeated imaging of a single animal ${ }^{11,22}$. Similarly, in vivo imaging can also provide invaluable insights into tumor cell mimicry whereby fluorescently tagged tumor cells can be imaged and tracked with an intra-vital CD31 antibody for endothelial cells to highlight the possibility of tumor cell transdifferentiation to directly form its own endothelium.

The versatility of the model is enhanced by the ability to image 4 fluorescent channels, providing an exhaustive number of combinations for the study of varying cellular and biological process. We are able to intravitally image CFP (blue), GFP (green), Cherry/RFP (red), and Alexa647/APC (far-red), simultaneously in a single field repeatedly over time. Researchers can genetically modify cells to express fluorochromes for each of the channels as well as purchase dyes and antibodies to highlight structural changes of particular interest. To date dyes and antibodies commonly used in studies include CD31 and dextran which both highlight the microvasculature and its changes in tissue ${ }^{1,11,23,24}$, although we utilized the far-red channel for this purpose both are available for use in the other channels mentioned. Similarly, other dyes including Sytox Orange, which will highlight areas of apoptosis, and markers such as those from the company Visen, have been specifically designed for use in vivo. Further to the four commonly used fluorescent channels mentioned, a second harmonic generation (SHG) channel can be added and optimized to image the endogenous collagen fibers of the model $^{7}$, visualizing the basement membrane surrounding vasculature.

To demonstrate the adaptability of our model, as well as the cell-cell interactions mentioned above, we have been able to study drug-cell interactions. We have looked at drug inhibitors like AMD3100, an SDF-1 inhibitor, and its role in signaling networks involved in the BMDC recruitment ${ }^{11}$. Similarly we have genetically engineered out U87 glioma xenografts cells to express VEGFTrap, a VEGF inhibitor ${ }^{25}$, in conjunction through an IRES to a GFP molecule. By using RFP+ BM we are able to study the role VEGF has on the recruitment of BMDCs to the vasculature. Most recently we have utilized the model to study drug kinetics looking at the mechanism behind the tumor-delineating drug Fluorescein ${ }^{26}$, at a cellular level. Through the use of a custom-built in house small animal irradiator we have been able to integrate stereotactic delivered radiation to assess the response of both tumor and BMDCs to treatment.

Through the use of our novel intravital imaging method researchers will gain insight into the single-cell real-time changes that occur in various pathologies and systems, aiding the elucidation of many functional and biological features of tissue changes.

\section{Protocol}

ALL ANIMAL WORK HAS BEEN CARRIED OUT UNDER AN ANIMAL CARE AND USE COMMITEE APPROVED PROTOCOL AND EXECUTED IN COMPLIANCE WITH ALL RELEVANT GUIDELINES, REGULATIONS AND REGULATORY AGENCIES.

For troubleshooting reference Table 2.

\section{Bone Marrow Reconstitution (Optional) Figure 1 (30 min prep, 5 min per mouse)}

All surgery should be performed using strict aseptic technique with autoclaved sterile equipment.

One donor mouse will reconstitute three host mice.

1. Anaesthetize recipient NODscid mice and position, with lead to shield the head, inside the center of the Gammacell 40 'exactor' irradiator.

2. Irradiate NODscid mice with $2.5 \mathrm{~Gy}$ total body irradiation (TBI).

CRITICAL STEP: Optimization of TBI may be needed due to variation in dose required for sufficient host immune cell depletion in different mouse strains.

Pause point: Host mice can be irradiated in advance but should be used within $24 \mathrm{hr}$ of irradiating.

3. Euthanize donor mice according to the institutional animal care committee guidelines. Clean the hindlimbs and remove the tibia and femur from both, stripping all excess tissue from the bones (Figures 1ai, 1aii).

CRITICAL STEP: Fibular bones are not viable for use due to very narrow lumen.

4. Remove end plates from both ends of four extracted bones and flush them out using a $22 \mathrm{G}$ needle and $1 \mathrm{ml}$ sterile PBS until they are white in appearance (Figure 1aiii). Reference Table 2.

5. Mix the extracted bone marrow (BM) suspension well and draw $300 \mu \mathrm{l}$ into three $27 \mathrm{G}$ tuberculin needles. Extracted BM should contain approximately $2 \times 10^{7}$ cells and is enough for 3 host mice reconstitutions. Reference Table 2.

6. Inject the suspension into the lateral tail vein of three previously irradiated NODscid mice from step 1.1 (Figure $1 \mathbf{b}$ ). Reference Table 2.

CAUTION: To check sterility of the BM injected we recommend plating the remaining BM and culture for $24 \mathrm{hr}$ to check for infection. Primary cause of death at this point is infection in the newly reconstituted mice. 


\section{Intracranial Window Generation - Figure 2 (30 min per mouse)}

All surgery should be performed using strict aseptic technique with autoclaved sterile equipment and under a heat lamp to keep animals warm (Figure 2A).

1. Anaesthetize recipient NODscid mice with IACUC approved anesthetic, Avertin IP injection at $0.5 \mathrm{mg} / \mathrm{g}$ and position, with lead to shield the head, inside the center of the Gammacell 40 'exactor' irradiator. Remove hair from the scalp. In addition apply tear gel to prevent corneal dehydration.

2. Clean scalp first with Betadine solution and then with alcohol, then make an incision from the midpoint of the ears to just above the eyes. Remove scalp $3 \mathrm{~mm}$ either side of the first incision, exposing the skull and landmarks of the skull surface (Figure 2Bi).

3. Elevate the periosteum by injecting $2 \%$ lidocaine:epinephrine solution and dissect away from skull surface (Figure 2Bii).

4. Using $2.7 \mathrm{~mm}$ trephine drill, weaken a $2.7 \mathrm{~mm}$ circle of the skull over the cortex of the right hemisphere between lambda and bregma. Do NOT penetrate the bone with the drill (Figure 2Biii). Reference Table 2.

CRITICAL STEP: if drill goes through the skull the brain surface will be damaged influencing results with additional trauma. In addition excessive bleeding will occur preventing a clear window from being generated.

5. Remove the weakened bone flap using dissection tweezers and dental hook and deliberate but controlled force (Figure 2Biii).

6. (Optional) If looking at tumor pathology, inject chosen tumor cell lines (with fluorescent reporter gene) into the center of the window generated in step 2.5 .

1. Load $10 \mu \mathrm{l} 30 \mathrm{G}$ Hamilton syringe with cell suspension and load needle into a stereotactic frame.

CRITICAL STEP: Cell number will need to be optimized for tumor cells in use and timeline of growth required. For U87 cultures we use $2 \times 10^{5}$ cells in $10 \mu$ l per mouse.

2. Load mouse onto the digital stereotactic frame align the needle to the center point of the window generated.

3. Lower needle until it just touches the cortical surface and reset the digital coordinates.

4. Lower the needle to $3.2 \mathrm{~mm}$ into the cortical tissue and inject at $3 \mathrm{~mm}$ deep.

5. Retract needle slowly then remove mouse from the frame.

CRITICAL STEP: Inject solution over the duration of $1 \mathrm{~min}$ and leave needle in position following injection to ensure reduced back flow. CRITICAL STEP: If minor hemorrhaging is encountered irrigate with sterile saline for 1-5 min to stop superficial bleeding. Proceed with subsequent steps.

7. Dampen brain surface with a drop of sterile PBS to keep the brain tissue irrigated.

8. Float a $3 \mathrm{~mm}$ coverslip onto brain surface to seal fully around the $2.7 \mathrm{~mm}$ window generated. Reference Table 2.

9. Dry surrounding skull bone then apply vetbond to the entire exposed skull to reseal scalp tissue to the skull bone and the coverslip in place. DO NOT apply an excess as it will leak under the glass and decrease the imaging potential.

10. Mix fresh dental acrylic powder and solution, roughly $30 \%(\mathrm{w} / \mathrm{v})$, and apply over the top of the vetbond. To ensure a tight seal overlap the acrylic slightly onto the glass coverslip edge (Figure 2Biv). Reference Table 2.

CAUTION: Dental acrylic is extremely hazardous so we recommend use of gloves and mask throughout the procedures.

CRITICAL STEP: Dental acrylic needs to remain pliable during molding to ensure a good seal and reduce excess. Build up of acrylic on the window will blur images.

11. Allow mice to recover in a warm cage.

\section{Stereotactic Radiation (Optional) - Figure 3 (25 min per mouse)}

Variations will exist in the different irradiators used and as such optimization will be required. We used a custom designed irradiator.

1. Anaesthetize mice using Isoflurane at $4 \%$ for induction followed by $1.5-2 \%$ throughout the procedure with $0.5-1$ liter $\mathrm{O}_{2}$ a min, and place onto custom head restrainer inside the stereotactic irradiator (Figure 3Ai).

2. Obtain a $360^{\circ}$ cone beam CT scan with the X-ray tube running at $40 \mathrm{kVp}$ and $0.05 \mathrm{~mA}$ through a $2 \mathrm{~mm}$ aluminum filter. Use the image to guide the movement of the stage, directing the radiation isocentre to the right hemisphere ensuring it is central in the Dorsal Ventral direction.

3. Insert the hemispheric collimator, $8 \mathrm{~mm} \times 11 \mathrm{~mm}$ block.

CRITICAL STEP: Collimators can be designed for many different setups and so can be enhanced to include and exclude different parts of the brain. $8 \mathrm{~mm} \times 11 \mathrm{~mm}$ defines a hemispheric area of the brain.

4. Obtain single CT orthogonal images both from top (AP) and bottom (PA) through the collimator to further enhance the placement of the brain, anatomical bone structures can be used for reproducibility.

5. Exchange the aluminum filter for a $0.93 \mathrm{~mm}$ copper treatment filter and administer half of the desired radiation dose with the $\mathrm{X}$-ray tube running at $225 \mathrm{kVp}$ and $13 \mathrm{~mA}$ from the top in an AP direction.

6. Return gantry to the bottom position and administer the second half of the radiation dose again with the $\mathrm{X}$-ray tube running at $225 \mathrm{kVp}$ and 13 $\mathrm{mA}$ form the bottom in a PA direction.

CRITICAL STEP: It is essential to irradiate from both the top and the bottom to reduce the RT gradient through the brain tissue, it also aids with the alignment of the isocentre to the center of the brain. 


\section{In vivo Two Photon Laser Microscopy - Figure 3 (1-3 hr per session)}

Variations will exist in the different microscopes used and as such optimization will be required. We used a Carl Zeiss LSM510 META Laser Scanning Confocal Microscope.

1. Anaesthetize mice with IACUC approved anesthetic, Avertin IP injection at $0.5 \mathrm{mg} / \mathrm{g}$ and clean window using alcohol spray.

2. A. (Optional) Inject tagged vascular dye 5-10 min prior to imaging, into the tail vein prior to imaging. Alexa647-Dextran used at 0.35 $\mu \mathrm{g} / \mathrm{g}$ or APC-CD31 used at $0.2 \mu \mathrm{g} / \mathrm{g}$. See Table 2.

B. (Optional) Inject Fluorescein via the tail vein at a dose of $7.7 \mathrm{mg} / \mathrm{kg}, 5 \mathrm{~min}$ prior to imaging, to delineate tumor. Reference Table 2.

3. Setup channels on the confocal microscope as determined by the fluorochrome used in the chimeric mouse generated. Table 1 demonstrates the channels described in this model.

4. Invert mouse onto the moveable microscope stage and stabilize the head in position with moldable plasticine. (Figure 3Aii) Reference Table 2.

CRITICAL STEP: The ICW needs to be perpendicular to the laser point and as such must be positioned horizontally to ensure the imaging is optimal.

5. Turn on first channel laser and use to position in the center of the ICW.

6. Use $5 x$ lens and take an image of the entire window to use as a map for further higher resolution images.

7. Imaging should be carried out using $10 \mathrm{X}$ and $20 \mathrm{X}$ 'long range' lenses to get the best image quality.

CRITICAL STEP: Channels and objectives on the 2PLM should be set up using engineered cells in vitro prior to the imaging of murine models to ensure they highlight the correct fluorochrome.

\begin{tabular}{|l|l|l|l|l|l|}
\hline Fluorochrome & CFP & $\begin{array}{l}\text { GFP / Fluorescein / } \\
\text { FITC }\end{array}$ & $\begin{array}{l}\text { mCherry / RFP / } \\
\text { DsRED }\end{array}$ & APC / Alexa647 & $\begin{array}{l}\text { SHG } \\
\text { Autofluorescence }\end{array}$ \\
\hline Excitation laser & $458 \mathrm{~nm}$ & $488 \mathrm{~nm}$ & $543 \mathrm{~nm}$ & $633 \mathrm{~nm}$ & $\begin{array}{l}\text { Chameleon laser } 820 \\
\mathrm{~nm}\end{array}$ \\
\hline Collection Filter & $480-520 \mathrm{~nm}$ & $500-550 \mathrm{~nm}$ & $565-615 \mathrm{~nm}$ & $650-710 \mathrm{~nm}$ & $390-465 \mathrm{~nm}$ \\
\hline
\end{tabular}

Table 1. Fluorochrome setup. Guides for the user to see the excitation laser and emission spectra collector used for each of the channels available in the model.

\section{Representative Results}

The optional step of reconstituting the BM of NODscid mice should result in an $80 \%$ uptake of the fluorescent 'donor' BM in $100 \%$ of the NODscid 'host' mice, however optimization of the TBI will be required to enhance the reconstitution in other non-immunocompromsed strains. If mice are unsuccessfully reconstituted they will get sick and die rapidly following the procedure, weakened mice may require additional food and water sources.

The ICW once finished should look like that seen in Figure 2Biv. It is ideal to have a ridge of acrylic surrounding the glass coverslip as this gives strength to the join with the skull. Perfect windows are reproducible and allow for repeated imaging for up to 8 weeks following their generation (Figure 2C). Images produced through these optimal windows will look as those seen in Figure 3Bi. Imperfections in the window generation will produce images of poor quality for example, air bubbles under the window will prevent whole fields of view being imaged, areas will appear dark as the air prevents the laser imaging (Figure 3Bii). With excess glue and acrylic on the coverslip there will be a high level of background fluorescence and areas of the field being blotted out as seen in Figure 3Biii, similarly if there is dirt on the window small dots of background fluorescence will be visible in the field (Figure 3Biv).

Success of ICW imaging is predetermined by the integrity of the surgical technique, however, even optimal ICWs can encounter problems during the imaging session. As such, a range and degree of clarity exists in the images generated as seen in Figure 3 . The publication quality image portrayed in Figure $\mathbf{3 C}$ occurs when everything is optimal. Although it is not possible for all mice, it is feasible to expect $80 \%$ of the images generated to look like this. One of the major flaws in the current microscopy setup is the use of inverted lasers. Mice have to be positioned on their backs and this results in excessive stress and discomfort that may lead to labored breathing during the imaging. This produces a 'lined' image as the breathing interferes with the averaging that occurs during the imaging, whereby, each pixel row is imaged four times and the average of the four is displayed in the final image. Any movement during the averaging, such as that caused by labored breathing, will create an artifact that appears as a line on the image, as seen in Figure 3D. Mice that are insufficiently anaesthetized during imaging may encounter this problem also. The 'lined' effect can be decreased by limiting the image averaging to on, however this will in turn reduce the image quality and may not eliminate the problem. Alternatively mice can be repositioned or retried when breathing has normalized. The use of an upright 2 PLM would negate this problem as mice could be imaged 'in the prone position'. An additional problem with imaging on an inverted 2PLM includes the limited accessibility for positioning the ICW once the mouse is on the microscope, and this leads to 'segmented' images like those seen in Figure 3E. Here the laser and coverslip are not positioned perpendicular to one another and as such the imaging occurs at an angle. This results in the generation of a 'segmented' image where the sides of the field of view are not imaged. The problem is easily solved with the repositioning of the mouse to ensure the coverslip is completely horizontal once on the head mount as seen in Figure 3Aii.

The model presented here was specifically used to examine the role of BMDCs in the vascularization of tumor tissue and demonstrates the ability to use three different channels simultaneously (Cherry, GFP, Far-red - Alexa647 and APC) making the CFP and SHG redundant for this particular story. We were able to image mice longitudinally for up to 8 weeks, studying the recruitment and integration of BM cells into the vasculature at a single cell level, with no detrimental effects caused by the window. This model demonstrates the ease of collecting dynamic 
information on the source and formation of vasculature and the interaction of different cell types of interest, previously lost through end point histological analysis. 


\begin{tabular}{|c|c|c|c|}
\hline Step & Problem & Reasoning & Solution \\
\hline 1.4 & Bone shatter & Blunt tools & $\begin{array}{l}\text { Collect bone marrow form a fresh } \\
\text { mouse using sharpened scissors } \\
\text { or fresh scalpel blade, bone } \\
\text { fragments will inhibit TV injection }\end{array}$ \\
\hline \multirow[t]{2}{*}{1.5} & Low extraction (low viscosity) & $\begin{array}{l}\text { Bone endplates cut too distally; } \\
\text { poor collection method }\end{array}$ & $\begin{array}{l}\text { Bones should be cut as proximally } \\
\text { as possible and collected with } \\
\text { the bone inside collection tube to } \\
\text { prevent splash back } \\
\text { Time should be spent to extract the } \\
\text { BM maximally and with care } \\
\text { If necessary pool more than one } \\
\text { mouse into } 1 \mathrm{ml}\end{array}$ \\
\hline & High Extraction (high Viscosity) & Low collection buffer & $\begin{array}{l}\text { Dilute out solution with extra } 0.1 \% \\
\text { BSA, split to three recipient mice } \\
\text { up to } 500 \mu \text { l per mouse maximum }\end{array}$ \\
\hline 1.6 & Bad Intravenous injection & $\begin{array}{l}\text { Poor vasodilation and vessel } \\
\text { visibility }\end{array}$ & $\begin{array}{l}\text { Enhance dilation with heat lamp. } \\
\text { Place mouse in tail vein restrainer } \\
\text { with built in light source to aid } \\
\text { access }\end{array}$ \\
\hline \multirow[t]{2}{*}{1} & Mice Sick & Infection & $\begin{array}{l}\text { Sacrifice mice according to } \\
\text { institution rules. Ensure tails are } \\
\text { cleaned prior to injection and check } \\
\text { sterility of extracted BM in culture }\end{array}$ \\
\hline & Mice die & Poor BM uptake & $\begin{array}{l}\text { Check \% of fluorescent BM uptake } \\
\text { of dead mouse. } \\
\text { Optimize TBI for strain of mice } \\
\text { being used } \\
\text { Increase amount of BM injection } \\
\text { (example use one donor mouse for } \\
\text { two recipients) }\end{array}$ \\
\hline \multirow[t]{2}{*}{2.4} & Minor hemorrhaging & Dura breached during drilling & $\begin{array}{l}\text { Pressure with gel pads and and } \\
\text { continuous sterile saline wash }\end{array}$ \\
\hline & Major hemorrhaging & Brain damaged by drilling & $\begin{array}{l}\text { Sacrifice mouse according to } \\
\text { institution guidelines }\end{array}$ \\
\hline 2.8 & Air-bubbles under coverslip & $\begin{array}{l}\text { Poor contact with cortical surface } \\
\text { prevents proper placement }\end{array}$ & $\begin{array}{l}\text { Remove coverslip and add extra } \\
\text { PBS to float the coverslip onto the } \\
\text { window to remove bubbles }\end{array}$ \\
\hline 2.10 & Slippage of coverslip during gluing & $\begin{array}{l}\text { Acrylic mass is heavy, placing } \\
\text { pressure on the coverslip and } \\
\text { moves the coverslip out of the way }\end{array}$ & $\begin{array}{l}\text { Tweezers should be used to hold } \\
\text { coverslip down while vetbond and } \\
\text { acrylic is applied }\end{array}$ \\
\hline 4.2 & Bad Intravenous injection & $\begin{array}{l}\text { Poor vasodilation and vessel } \\
\text { visibility }\end{array}$ & $\begin{array}{l}\text { Enhance dilation with heat lamp. } \\
\text { Place mouse in tail vein restrainer } \\
\text { with built in light source to aid } \\
\text { access }\end{array}$ \\
\hline $\begin{array}{l}4.4 \\
\text { Figure 3C }\end{array}$ & 'Lined' images & Labored or irregular breathing & $\begin{array}{l}\text { Remove mouse from frame and } \\
\text { allow to recover } \\
\text { Increase level of anaesthetic } \\
\text { administered and adjust position to } \\
\text { ensure neck is not overly flexed or } \\
\text { extended to prevent breathing }\end{array}$ \\
\hline \multirow[t]{3}{*}{ Figure 3C } & 'Segmented' image & Brain is not parallel to objectives & $\begin{array}{l}\text { Adjust position of coverslip to } \\
\text { ensure it is flat }\end{array}$ \\
\hline & Vessel not visualized & injection not seen intravascularly & $\begin{array}{l}\text { Redo injection into alternative tail } \\
\text { vein, warm tail to ensure good } \\
\text { vasodilation }\end{array}$ \\
\hline & High background & $\begin{array}{l}\text { Dirty coverslip, } \\
\text { Air Bubbles, Acrylic }\end{array}$ & $\begin{array}{l}\text { Wipe coverslip with damp } 70 \% \\
\text { ethanol cloth, do not soak as } \\
\text { may penetrate under acrylic and } \\
\text { damage the brain tissue }\end{array}$ \\
\hline
\end{tabular}


Table 2. Troubleshooting. A guideline for corrective steps required for problematic areas of the procedures.

Model A - Intracranial Window Generation

Imaging and Sacrificing Plan

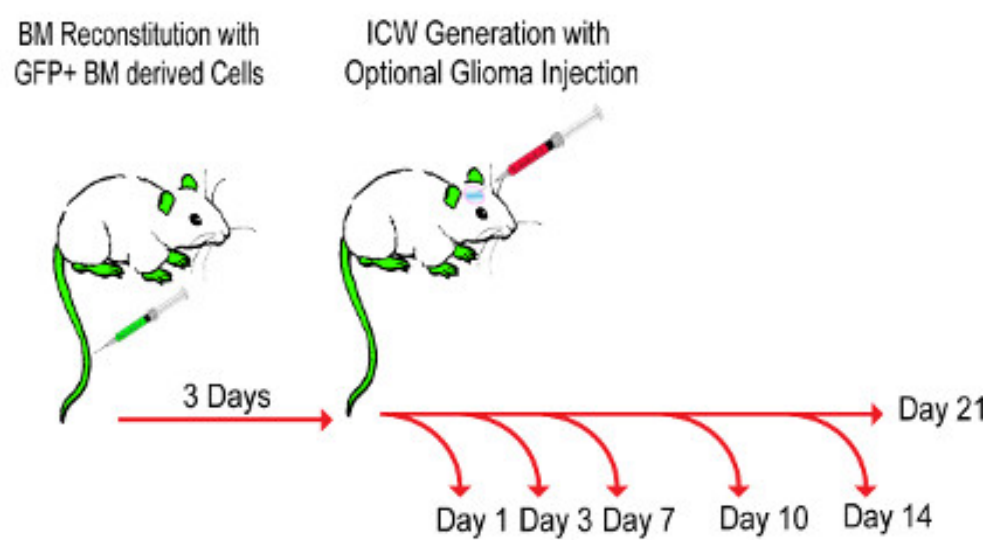

\section{Model B - Intracranial Window Generation + Treatment}

Imaging and Sacrificing Plan

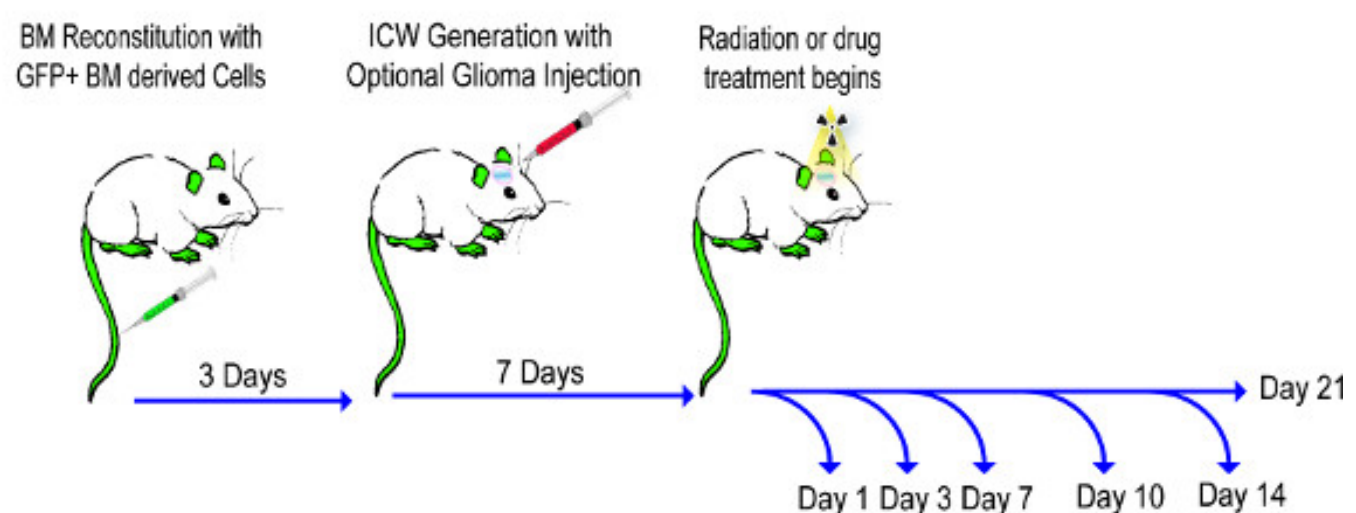

Schemata 1. Experimental flow diagram. This demonstrates the timeline of events through experimental steps 1-4. Mouse models are setup over a week, to ensure the BM reconstitutes properly, and are not treated with drug until day 7 of the tumor to ensure the tumor grafts. Click here to view larger figure. 

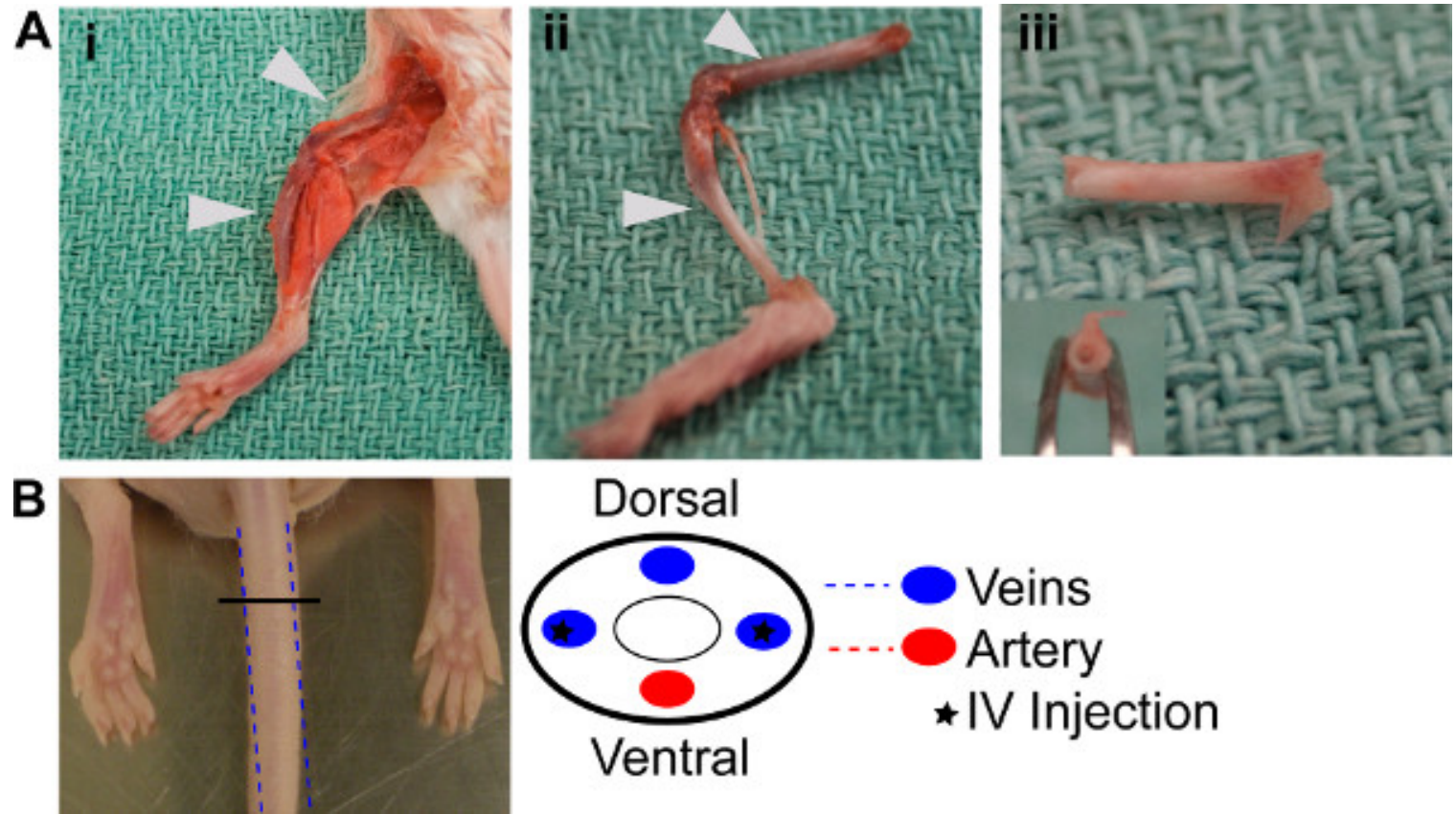

Figure 1. BM Reconstitution.(A) BM extraction procedure i. Dissection of the hind limb bones. ii. Dissected femur and tibia from the hind limbs, cleaned and ready for extraction. iii. Bones with end plate removed and flushed through, demonstrating the white appearance of the empty bones. (B) Schemata demonstrating where lateral veins for injection are positioned in the tail.

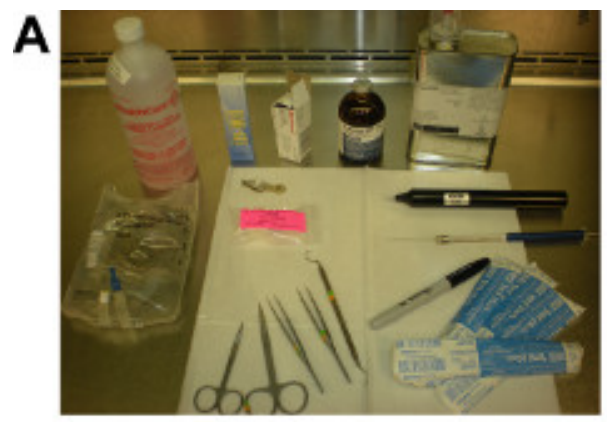

B

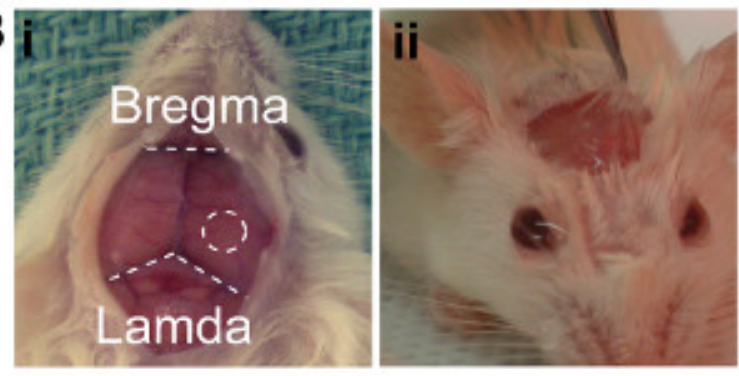

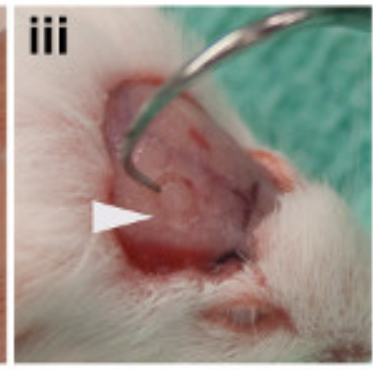
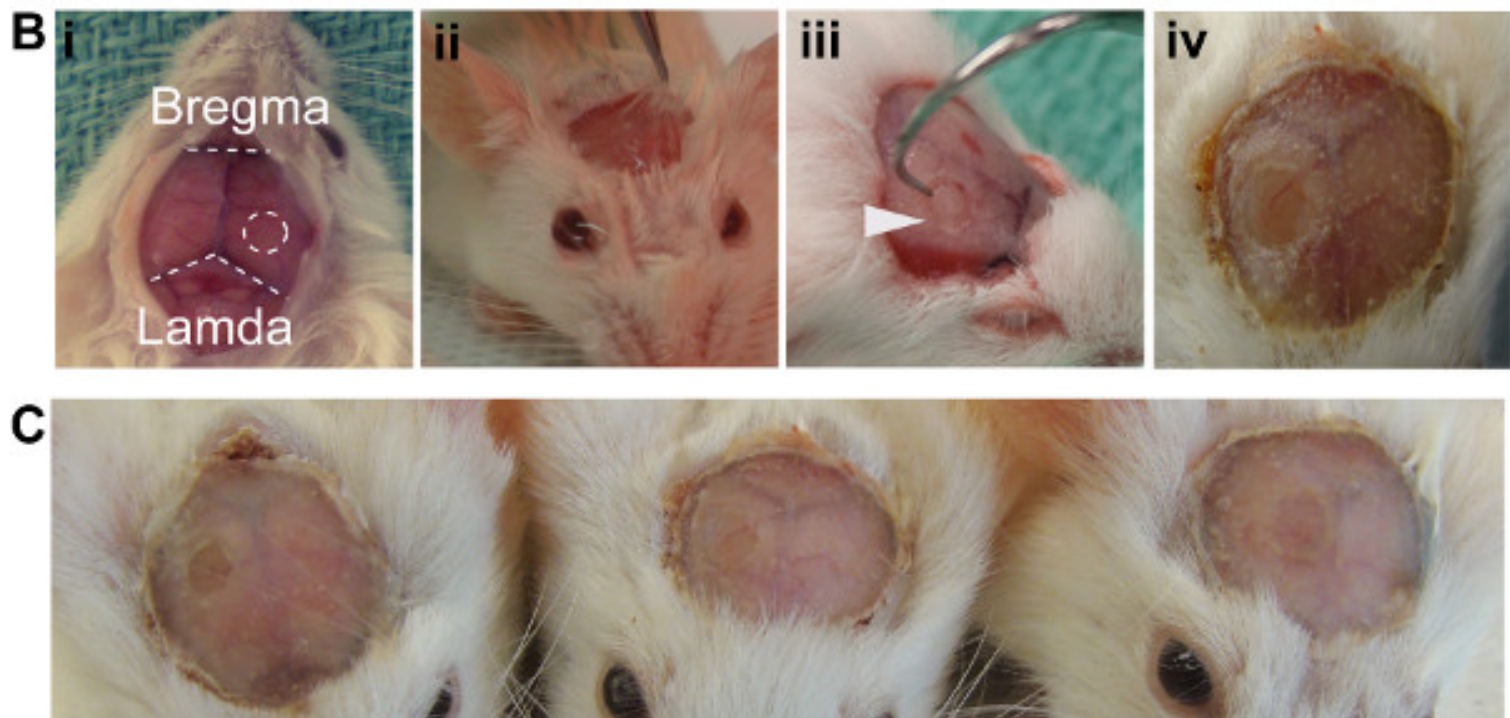

Figure 2. ICW generation. (A) Aseptic setup recommended (B) i. Exposed skull surface reveals the landmarks required for surgery, ICW should be positioned on the right hemisphere equidistant from the bregma and lamda. ii. Periostieum lifted with lidocaine solution, ready for removal. iii. Dental hook required for the removal of the bone fragment generated with the drill. iv. Finished ICW with dental acrylic. (C) Three example windows demonstrate the reproducibility of the method. 
A i

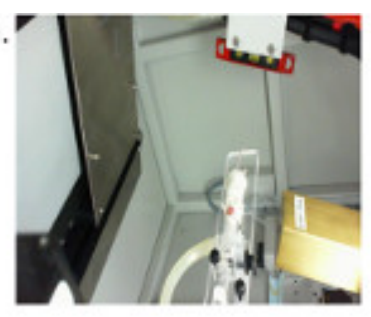

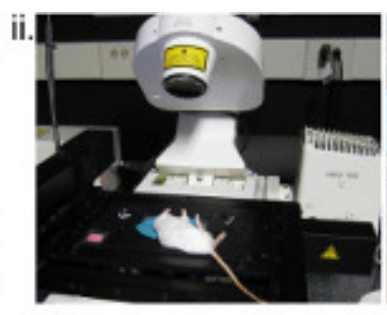

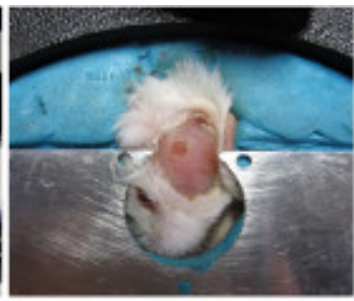

B

B i. Perfect

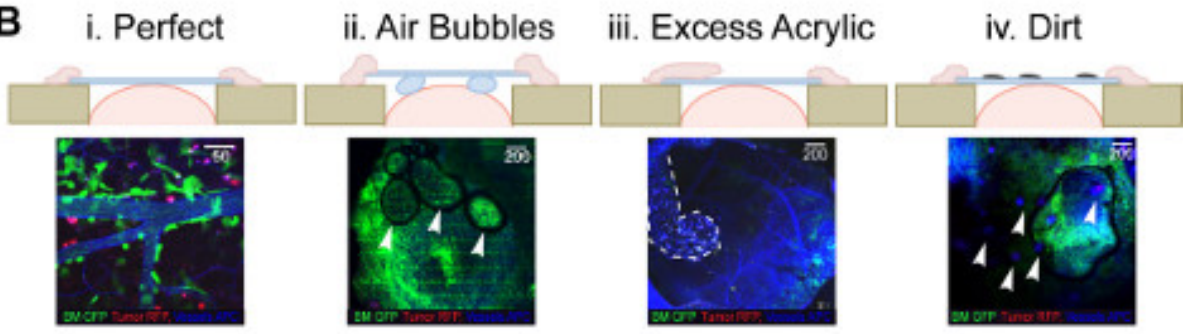

iii. Excess Acrylic

iv. Dirt

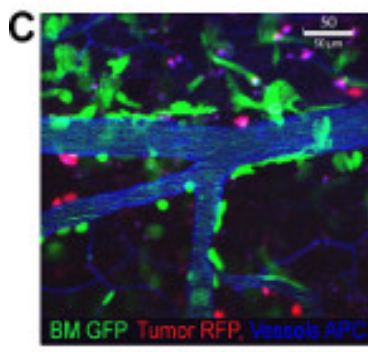

Perfect Image

Perpendicular Lens No breathing artifact

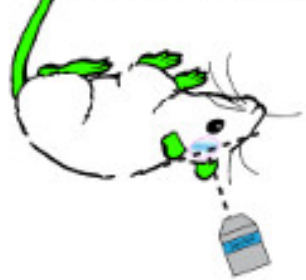

Figure 3. 2PLM anticipated results. All images show green BM, red tumor, Blue (pseudo colored far red) vasculature. (A) i. Demonstrates the head frame which retains isoflurane flow inside the small animal irradiator. The $8 \times 11 \mathrm{~mm}$ collimator can also be seen moving around through the gantry. ii. Mouse in the inverted positioned in the head frame required for imaging, moldable plastercine ensures all windows can be accommodated. (B) Demonstrative photos of the outcomes of problems with the window generation from i. an optimal window, ii. a window with air bubbles trapped underneath, iii acrylic spillage over the coverslip and iv. dirt on the window itself. (C) Highlights the problems that occur with imaging following successful generation of an ICW i. optimal imaging ii. breathing artefacts create a 'lined' image iii. coverslip not perpendicular to the laser generate a 'segmented' image. Click here to view larger figure. 
A
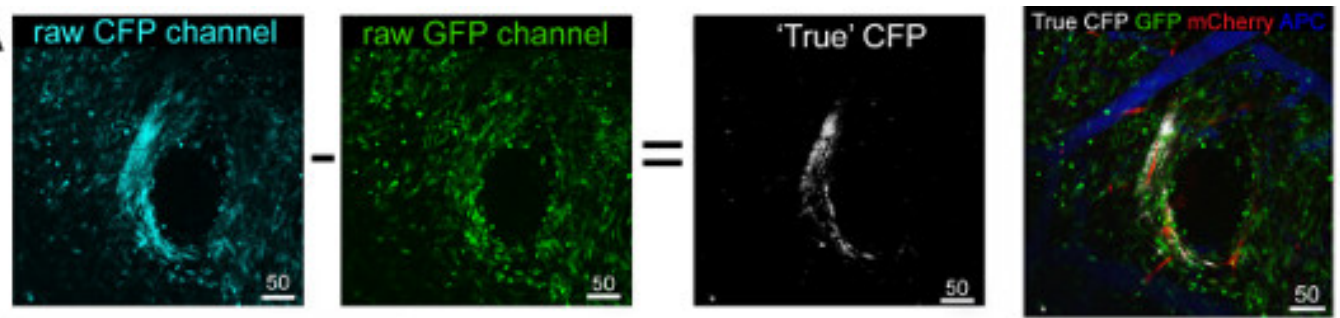

B

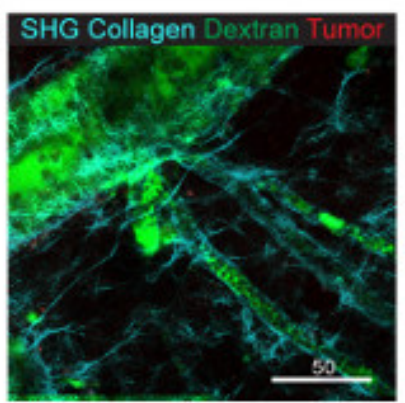

C
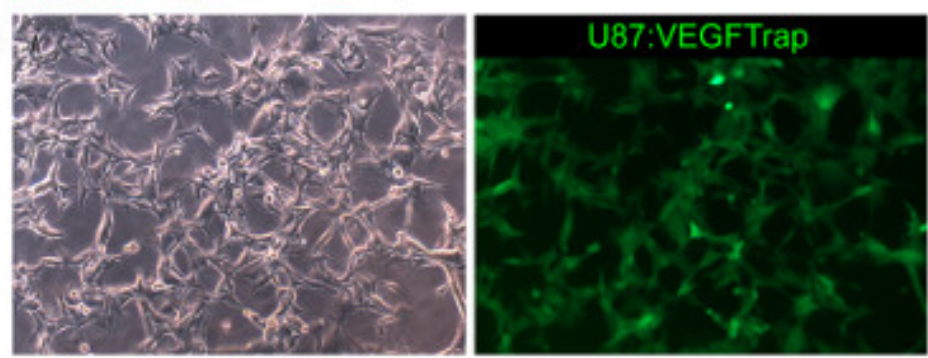

In vitro

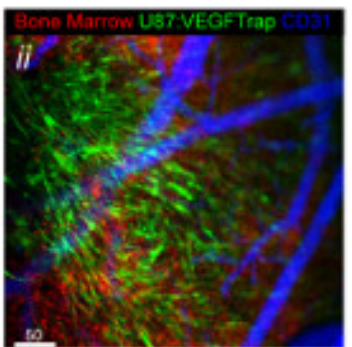

In vivo
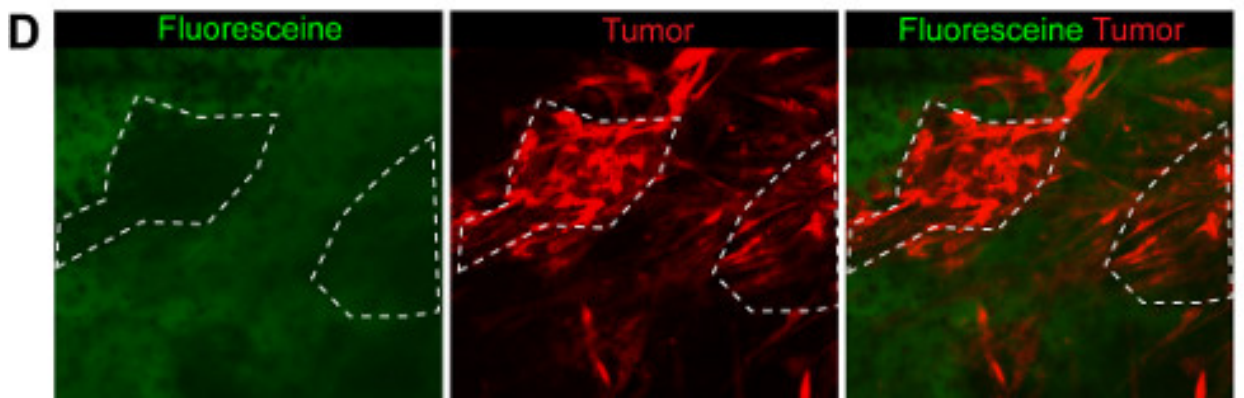

Figure 4. Functional uses and adaptations of the Model. (A) CFP post imaging processing whereby GFP image is subtracted from CFP image to reveal the true CFP image that can be overlayed the other three channels in white. Green BM, Red tumor, White CSCs, Blue (pseudo colored far red) vasculature (B) Demonstration of the collagen fibers that can be imaged with SHG. Green Dextran, red BM, Cyan Collagen (C) i. VEGFTrap cells in vitro demonstrate the GFP signal produced with the VEGFtrap. ii. In vivo imaging demonstrates the VEGFtrap cells clearly and in addition highlights the ease of switching channels to demonstrate the system you are looking at. Green VEGFTRap + Tumor, Red BM, Blue (pseudo colored far red) vasculature. (D) Demonstrates the collection of Fluorescein in the stroma of the tumor and not in the cells directly, shown by the lack of green signal overlay with red tumor. Green Fluorescein, Red tumor. Click here to view larger figure.

\section{Discussion}

The three channels described in all the images so far are interchangeable for three markers of interest in other models and leaves researchers with an invaluable set of tools to look an numerous cell types and interactions. Planning is required to ensure all markers and cell types have successfully integrated a reporter fluorescence molecule in a distinct channel.

In addition to the standard three channels used here we were also able integrate a fourth CFP channel (Figure 4A) and an SHG based collagen channel ${ }^{7}$ (Figure 4B). This extends the possibility of looking at cellular interactions in vivo and in addition allows the user to undertake population mixing to study specific cell-cell interactions while retaining two channels for other markers. For example, we have looked at tumor cells (RFP) and cancer stem cells (CFP) at a ratio of 1:3 with an interest in comparing their intratumoral interactions (Figure 4A). We observed that 7 days post implantation of the mixed population the ratio was upheld and both cell populations could still be seen.

The CFP channel provides technical issues due to the overlap with the GFP channel in both excitation and emission spectra and as such requires post imaging processing to define the true CFP+ cells from those that are actually GFP+. Post imaging processing can be carried out on the 2-photon microscopes built in Zeiss LSM software directly whereby, the GFP image is subtracted from the CFP image resulting in the 
true CFP cells being left behind (Figure 4A). The premise for the ratio subtraction is dependent on equally bright images and is due to the CFP laser $(458 \mathrm{~nm})$ fluorescing both CFP and GFP cells whilst the GFP laser $(488 \mathrm{~nm})$ is too high to fluoresce the CFP cells. In addition to CFP we have also been able to use previously published setups to look at the SHG levels and so depict the collagen fibers which make up the basement membrane surrounding vasculature, (Figure 4B).

Another adaptation of this model has taken advantage of a custom built small animal irradiator which has the ability to use stereotactic guided radiation to irradiate sections of tissue as small as $2 \mathrm{~mm}$ in diameter. By targeting the window with irradiation it is possible to look at the effect radiation has on the underlying tissue. We have recently published a study looking at the effect radiation has on normal brain tissue with respect to the recruitment of BMDCs to the vasculature, looking specifically at their role in post radiation tissue changes. We found that the recruitment of the BMDCs was both time and dose dependent in normal tissue ${ }^{11}$.

It is feasible to study the mechanisms of delivery and integration of therapeutics providing the drug is tagged with a fluorescent marker. During our research we have been able to track the production of VEGFTrap, an anti-angiogenic drug which blocks all VEGF signaling in the local area of production ${ }^{25}$. By genetically modifying our tumor cells to express the VEGFtrap gene coupled with an IRES to EGFP (Figure 4Ci) and using RFP 'donor' BM we were able to image the EGFP:VEGFtrap production, BMDC interaction and vasculature simultaneously (Figure 4Cii). This demonstrated the versatility of the model and channels available. It is also feasible to look at kinetics of the drug due to the promise of single-cell resolution. Fluorescein (GFP+) is used to delineate tumors during surgery to ensure maximal resection is achieved ${ }^{26}$. By injecting intravenously whilst imaging it is possible to demonstrate that the delineation occurs NOT through the active uptake of the Fluorescein into the tumor cells themselves, but instead through the collection of the drug into the stromal area with time, seen by the lack of co-localization 10 min following the injection of the Fluorescein (Figure 4D).

Overall our strategy combines novel and existing techniques to achieve a unique experimental platform, which is advantageous in the study of dynamic cellular interactions. This strategy has proven to be an invaluable approach for examining high-resolution single-cell dynamic evolution of BMDC, tumor and normal brain vascularity in response to therapy, intracranially. Intravital imaging can provide a better understanding of the molecular regulators of BMDC recruitment, migration and differentiation in intracranial brain tumor vasculature as well as many other dynamic processes adaptable to other research fields. Inhibiting putative factors that regulate BMDC in combination with other therapeutic strategies can aid the identification of the precise timing of combinatorial therapies. Furthermore, this strategy can be adapted to many future projects utilizing not only in vivo intravital staining dyes, but also small molecular inhibitors and nanoparticles that are fluorescently tagged allowing researchers to trace the distribution and migration patterns of more targeted therapeutics as well as look longitudinally at their kinetics.

\section{Disclosures}

Authors have nothing to disclose.

\section{Acknowledgements}

We would like to thank the Advanced Optical Microscopy Facility at the princess Margaret hospital, in particular James Jonkman for their assistance in the initial setup of channels on the 2Photon microscope. The authors would like to acknowledge the Spatio-Temporal Targeting and Amplification of Radiation Response (STTARR) program and its affiliated funding agencies. We thank Dr. Peter Tonge, Dr.lacovos Michael and Dr.Andras Nagy for supplying the VEGFTrap plasmids and for their manuscript corrections and feedback. The continued support and discussion from staff at the BTRC has been invaluable and we would like to Commemorate Dr.Abhijit Guha for his scientific input. Work was funded by ClHR and NIH grants.

\section{References}

1. Kienast, Y., et al. Real-time imaging reveals the single steps of brain metastasis formation. Nature Medicine. 16 116-122 (2010).

2. Holtmaat, A., et al. Long-term, high-resolution imaging in the mouse neocortex through a chronic cranial window. Nat. Protoc. 4, 1128-1144 (2009).

3. Hansen-Algenstaedt, N., et al. Long-term observation reveals time-course-dependent characteristics of tumour vascularisation. Eur. J. Cancer. 41, 1073-1085 (2005).

4. Kherlopian, A.R., et al. A review of imaging techniques for systems biology. BMC Systems Biology. 2, 74 (2008).

5. Kedrin, D., et al. Intravital imaging of metastatic behavior through a mammary imaging window. Nature Methods. 5, 1019-1021 (2008).

6. Perentes, J.Y., et al. In vivo imaging of extracellular matrix remodeling by tumor-associated fibroblasts. Nature Methods. 6, 143-145 (2009).

7. Brown, E., et al. Dynamic imaging of collagen and its modulation in tumors in vivo using second-harmonic generation. Nature Medicine. 9, 796-800 (2003).

8. Marker, D.F., Tremblay, M.-E., Lu, S.-M., Majewska, A.K., \& Gelbard, H.A. A Thin-skull Window Technique for Chronic Two-photon In vivo Imaging of Murine Microglia in Models of Neuroinflammation. J. Vis. Exp. (43), e2059, doi:10.3791/2059 (2010).

9. Drew, P.J., et al. Chronic optical access through a polished and reinforced thinned skull. Nature Methods. 7, 981-984 (2010).

10. Yang, G., Pan, F., Parkhurst, C.N., Grutzendler, J., \& Gan, W.-B. Thinned-skull cranial window technique for long-term imaging of the cortex in live mice. Nature Protocols. 5, 201-208 (2010).

11. Burrell, K., Hill, R.P., \& Zadeh, G. High-resolution in-vivo analysis of normal brain response to cranial irradiation. PLoS ONE. 7, e38366 (2012).

12. Tate, M.C. \& Aghi, M.K. Biology of angiogenesis and invasion in glioma. NURT. 6, 447-457 (2009).

13. Aghi, M. \& Chiocca, E.A. Contribution of bone marrow-derived cells to blood vessels in ischemic tissues and tumors. Mol. Ther. 12, $994-1005$ (2005).

14. Nussenbaum, F. \& Herman, I.M. Tumor angiogenesis: insights and innovations. J. Oncol. 2010, 132641 (2010). 
15. Zhang, H.-r., et al. Incorporation of endothelial progenitor cells into the neovasculature of malignant glioma xenograft. J. Neuroonco. 93, 165-174 (2009).

16. Blouw, B., et al. The hypoxic response of tumors is dependent on their microenvironment. Cancer Cell. 4, 133-146 (2003).

17. Du, R., et al. HIF1alpha induces the recruitment of bone marrow-derived vascular modulatory cells to regulate tumor angiogenesis and invasion. Cancer Cell. 13, 206-220 (2008).

18. Rajantie, l., et al. Adult bone marrow-derived cells recruited during angiogenesis comprise precursors for periendothelial vascular mural cells. Blood. 104, 2084-2086 (2004).

19. Shinde Patil, V.R., et al. Bone marrow-derived lin(-)c-kit(+)Sca-1+ stem cells do not contribute to vasculogenesis in Lewis lung carcinoma. Neoplasia. 7, 234-240 (2005).

20. Purhonen, S., et al. Bone marrow-derived circulating endothelial precursors do not contribute to vascular endothelium and are not needed for tumor growth. Proc. Natl. Acad. Sci. U.S.A. 105 6620-6625 (2008).

21. Aghi, M., Cohen, K.S., Klein, R.J., Scadden, D.T., \& Chiocca, E.A. Tumor stromal-derived factor-1 recruits vascular progenitors to mitotic neovasculature, where microenvironment influences their differentiated phenotypes. Cancer Research. 66, 9054-9064 (2006).

22. Burrell, K. \& Zadeh, G. Molecular Mechanisms of Tumor Angiogenesis., InTech, (2012).

23. Thorball, N. FITC-dextran tracers in microcirculatory and permeability studies using combined fluorescence stereo microscopy, fluorescence light microscopy and electron microscopy. Histochemistry. 71, 209-233 (1981).

24. Tolentino, M.J., et al. Angiography of fluoresceinated anti-vascular endothelial growth factor antibody and dextrans in experimental choroidal neovascularization. Arch. Ophthalmol. 118, 78-84 (2000).

25. Holash, J., et al. VEGF-Trap: a VEGF blocker with potent antitumor effects. Proceedings of the National Academy of Sciences of the United States of America. 99, 11393-11398 (2002).

26. Shinoda, J., et al. Fluorescence-guided resection of glioblastoma multiforme by using high-dose fluorescein sodium., 1-7 (2003). 